One-pot synthesis of small and uniform gold nanoparticles in water by

\title{
flash nanoprecipitation
}

Tianyi Tu, Wenjuan Zhou, Mingwei Wang, Xuhong Guo, Li Li,* Martien A. Cohen Stuart and Junyou Wang*

State-Key Laboratory of Chemical Engineering, and Shanghai Key Laboratory of Multiphase Materials Chemical Engineering, East China University of Science and Technology, Shanghai 200237, People's Republic of China

*To whom correspondence should be addressed:

E-mail: junyouwang@ecust.edu.cn, lili76131@ecust.edu.cn

This file includes:

1. DLS analysis of the AuNPs size and size distribution (Figure S1)

2. Calculation of Reynolds number (Table S1)

3. Preparation of AuNPs by normal mixing and MIVM (Figure S2)

4. UV-vis spectra of AuNPs coated with different capping agents (Figure S3) 

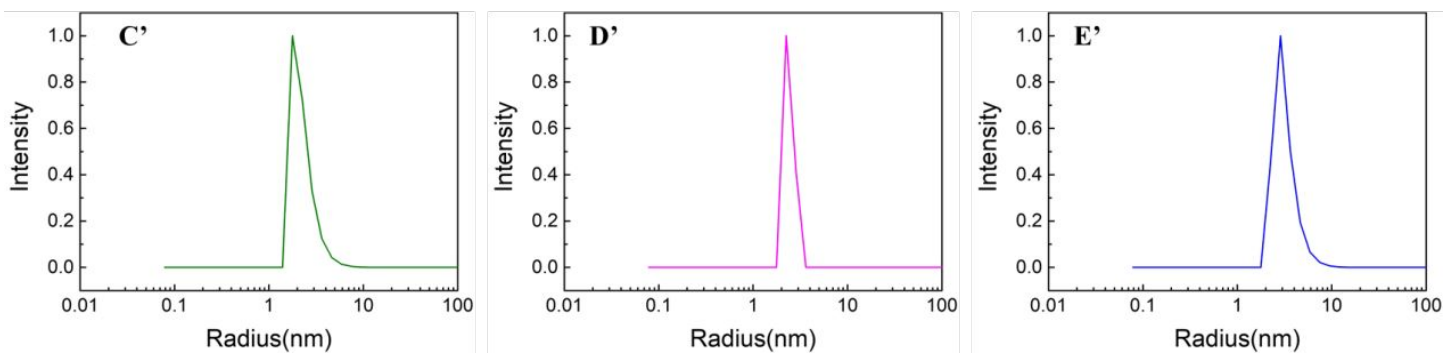

Figure S1. Size and size distribution of AuNPs analyzed by DLS. C', D' and E' correspond to AuNPs in Figure1 C, D and E. Number weighted CONTIN fit is applied for processing the date and analysis of the particle size ${ }^{1}$. 


\section{Calculation of Reynolds number}

The Reynolds number $(\mathrm{Re})^{2}$, a ratio of inertial force to viscous force, was used to quantify the mixing.

$$
\operatorname{Re}=\frac{\rho V D}{\eta}=\frac{\rho Q D}{s \eta}
$$

where $\rho$ is a fluid density, $\eta$ is a fluid viscosity, $V$ is a velocity, and $Q$ is a flow rate, D is a diameter of an inlet nozzle, and s is a cross sectional area of an inlet nozzle.

All experiments were done with four inlet MIVM. All mixer inlets were connected to plastic syringes $(20 \mathrm{~mL})$ via Teflon tubing with $1.5 \mathrm{~mm}$ ID. Four syringes contained different materials with different concentration. However, the material concentration in aqueous solution is very low. In this study, we assumed the density and viscosity of these material flow are the same as that of water.

$$
\operatorname{Re}=\sum_{i=1}^{4} \operatorname{Re}_{i}=\sum_{i=1}^{4} \frac{\rho_{i} Q_{i} D_{i}}{\mathrm{~s}_{i} \eta_{i}}
$$

where $\rho_{\mathrm{i}}$ is the density of the $i$ th component, $\mathrm{V}_{\mathrm{i}}$ is a velocity of the $i$ th component, $\mathrm{D}_{\mathrm{i}}$ in this study is the diameter of the $i$ th inlet nozzle $\left(\mathrm{D}_{\mathrm{i}}=1.1 \times 10^{-3} \mathrm{~m}\right), \mathrm{s}_{\mathrm{i}}$ is the cross sectional area of the $i$ th inlet nozzle $\left(1.65 \times 10^{-6} \mathrm{~m}^{2}\right.$ for all nozzles in the mixer used herein), and $\eta_{\mathrm{i}}$ is the viscosity of the ith component ${ }^{3}$. This study assumed $\rho_{\mathrm{i}}=1.0 \times 10^{3} \mathrm{~kg} \cdot \mathrm{m}^{-3}$ and $\eta_{\mathrm{i}}=8.9 \times 10^{-4} \mathrm{~Pa} \cdot \mathrm{s}$ at room temperature.

In this study, the velocity was changed from $1 \mathrm{~mL} / \mathrm{min}$ to $40 \mathrm{~mL} / \mathrm{min}$, the Reynolds number was calculated in the table. 
Table S1. Reynolds number of MIVM mixing at different injection rate

\begin{tabular}{|c|c|}
\hline Flow rate (mL/min) & Reynolds number \\
\hline 1 & 49.9 \\
\hline 4 & 199.8 \\
\hline 10 & 499.4 \\
\hline 30 & 1498.1 \\
\hline 40 & 1997.5 \\
\hline
\end{tabular}

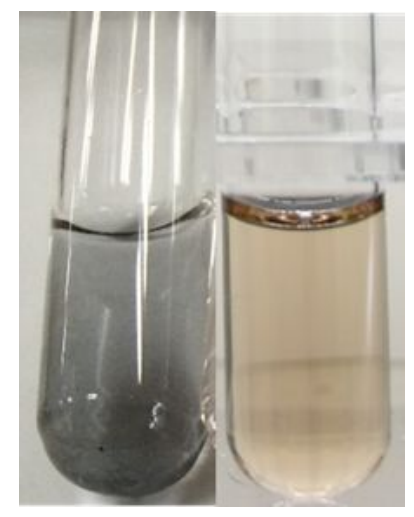

Figure S2. AuNPs prepared by normal mixing in beaker (left) and MIVM (right). All the chemicals were at same concentrations. 


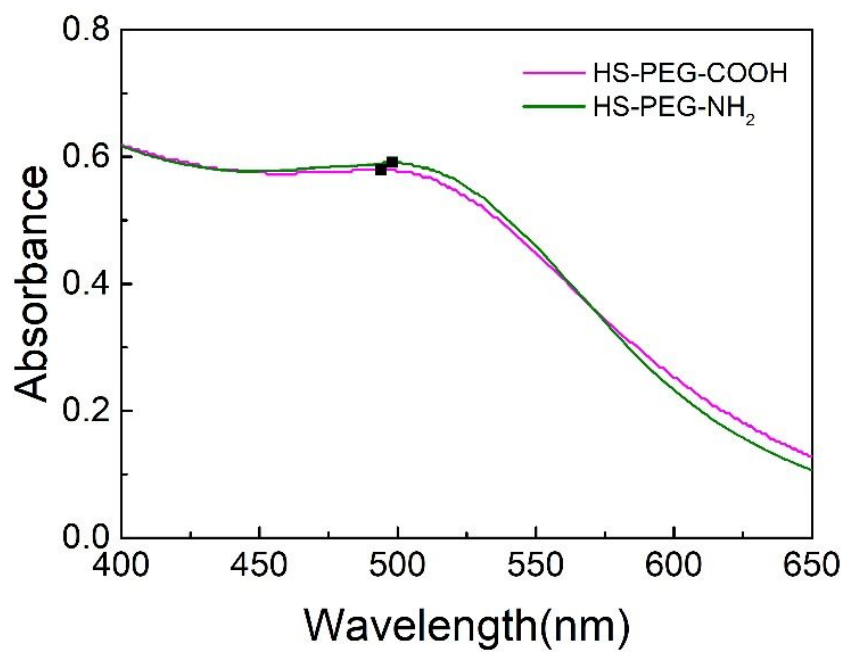

Figure S3. UV-vis spectra of AuNPs coated with different capping agents (HS-PEG-

$\mathrm{COOH}$, HS-PEG-NH $\mathrm{N}_{2}$ ) prepared at same condition (HAuCl4: 1.25mM, NaBH4:

12.5mM, HS-PEG-R: $0.0625 \mathrm{mM}$ ) 


\section{References:}

(1) Jan Bart ten Hove, L. M. I. S., Junyou Wang, Aldrik H. Velders, Size-controlled and water-soluble gold nanoparticles using UV-induced ligand exchange and phase transfer. Chem. Commun. 2018, 54, 13355-13358.

(2) Zhu, Z. X., Flash Nanoprecipitation: Prediction and Enhancement of Particle Stability via Drug Structure. Mol. Pharmaceut 2014, 11, 776-786.

(3) Zhu, Z. X., Effects of amphiphilic diblock copolymer on drug nanoparticle formation and stability. Biomaterials 2013, 34, 10238-10248. 